\title{
Assessing the Potential of Thermal Imaging in Recognition of Breast Cancer
}

\author{
Hossein Ghayoumi Zadeh ${ }^{*}$, Javad Haddadnia ${ }^{1}$, Nasrin Ahmadinejad ${ }^{2}$, \\ Mohammad Reza Baghdadi ${ }^{3}$
}

\begin{abstract}
Background: Breast cancer is a common disorder in women, constituting one of the main causes of death all over the world. The purpose of this study was to determine the diagnostic value of the breast tissue diseases by the help of thermography. Materials and Methods: In this paper, we applied non-contact infrared camera, INFREC R500 for evaluating the capabilities of thermography. The study was conducted on 60 patients suspected of breast disease, who were referred to Imam Khomeini Imaging Center. Information obtained from the questionnaires and clinical examinations along with the obtained diagnostic results from ultrasound images, biopsies and thermography, were analyzed. The results indicated that the use of thermography as well as the asymmetry technique is useful in identifying hypoechoic as well as cystic masses. It should be noted that the patient should not suffer from breast discharge. Results: The accuracy of asymmetry technique identification is respectively $91 / 89 \%$ and $92 / 30 \%$. Also the accuracy of the exact location of identification is on the $61 / 53 \%$ and 75\%. The approach also proved effective in identifying heterogeneous lesions, fibroadenomas, and intraductal masses, but not ISO-echoes and calcified masses. Conclusions: According to the results of the investigation, thermography may be useful in the initial screening and supplementation of diagnostic procedures due to its safety (its non-radiation properties), low cost and the good recognition of breast tissue disease.
\end{abstract}

Keywords: Asymmetry technique - biopsy - breast cancer - thermography

Asian Pac J Cancer Prev, 16 (18), 8619-8623

\section{Introduction}

Breast cancer is a common phenomenon in women. It is also one of the main causes of cancer death in women all over the world (Kelly et al., 2010). The most comprehensive statistical data on the prevalence of this disease has been established by the Center of diseases Control and Prevention.This data portrays a dramatic increase in breast cancer during the last 50 years in the United States and After 30 years, a sudden increase occured in the rate of breast cancer, in addition to the short steady state that occurs during the ages of 45 and 50 years (Saika and Sobue, 2009). Furthermore, the World Health Organization (WHO) has estimated that in 2030, the rate of cancer in the world will get to 27 million people (Araujo et al., 2014). The most widespread type of cancer in women is breast cancer, it is also the second or the third and most common malignancy in developing countries(Acharya et al., 2012). There are several diagnostic methods for the detection of breast cancer. Early diagnosis is important for successful treatment. Due to the direct relationship between the delay of the early detection of the cancer and death resulting from it, the method that has outstanding characteristics such as being available, reliable and noninvasive which is of immense importance in this regard. Therefore, the use of additional diagnostic techniques such as thermography can be highly beneficial for patients (Lahiri et al., 2012). Abnormal thermal patterns can be detected easily by means of thermal imaging. Generally, the findings of temperature measurement in comparison with other clinical findings are considered as a possible ways to evaluate the possible correlation. Although it is non-specific method, sometimes it depends on the severity of the background and the surrounding environment, but there are several reasons that have caused the thermal imaging to achieve wide recognition in the medical community. First of all, thermal imaging is a remote, non-contact and non-invasive method ( $\mathrm{Ng}, 2009)$. The Imaging Time is very limited; therefore, it is possible to monitor a large number of people simultaneously. Rendition of the Thermogram colors is by no doubt easy and fast. Additionally, this method can record only natural radiation from the surface of the skin. And there is not any evidence from harmful rays. Therefore, it is

${ }^{1}$ Biomedical Engineering Department, Hakim Sabzevari University, Sabzevar, ${ }^{2}$ Advanced Diagnostic and Interventional Radiology Research Center, Tehran University of Medical Sciences, ${ }^{3}$ Research Institute for Information and Communication Technology, Tehran, Iran*For correspondence: h.ghayoumizadeh@gmail.com 
suitable for a long term and repeated use.Finally; thermal imaging is a real-time method which can monitor dynamic changes of temperature. The Radiation Potential for human dark skin is more or less equal to the constant value of $0.01+0.98$ between wavelength 2 and 14 micrometers (Watmough et al., 1970). Therefore, in this range of wavelength, human skin acts as a black physical body, and due to its high absorption coefficient of $2 / 5$ to $1 / 3 \mathrm{~mm}$ at wavelengths between $2 / 2$ and 5 micrometers, thermal radiation results from the outer surface of the skin(Steketee, 1973). Asymmetry Techniques are among the basic principles and methods for analyzing thermal images. Authors in (4) suggested that they applied an approach based on image segmentation by adopting image processing techniques such as Hough transform for the analysis of asymmetry. Analysis of asymmetry was grounded in the changes of temperature, skewness and kurtosis. Recently, according to the conducted studies in other countries, it has been shown that Thermography imaging system gives the correct answer for the diagnosis of health or disease, by taking appropriate diagnostic and correct answers. Generally, it would be appropriate office along with other methods as a complementary approach (Nicandro et al., 2013; Vreugdenburg et al., 2013; Collett et al., 2014). In the experiment performed on 92 patients suffering from cancer, Biopsy testing was conducted and the obtained Value of sensitivity was $97 \%$ (Arora et al., 2008). In a survey conducted in 2010, which was performed on 50 patients, the value of sensitivity was $78 \%$ and value of specificity was $75 \%$ (Wishart et al., 2010). In a study conducted on 49 patients with cancer, the value of sensitivity was $97 \%$, and the value of specificity was $99 \%$.The results of this study showed that breast cancer screening by means of thermography is a very useful tool (Muffazzal et al., 2014). Notwithstanding, the relative achievement of mammography, there is a need for ongoing research to increase the sensitivity of breast cancer recognition, especially younger women. Albeit, mammography is currently considered to be the "golden standard" technology for the diagnosis of breast tumors , the performance of this approach is less used in younger women. Because of the complexity of the imaging of the compact breast tissue and film interpretation. The main objective of the present study is to determine the usability and functionality of thermography system to identify some of the breast tissue disease compared with the ultrasound system.

\section{Materials and Methods}

The present study has adopted thermo- imaging that utilizes thermography system with non -contact infrared camera (InfReC R500 model). The Scheme of its image is shown in Figure 1.

Features of infrared camera R500 are shown in Table 1.

In thermography, the patient was placed in a certain distance, which was approximately $40 \mathrm{~cm}$ from the infrared camera. It was noted that the emitted Waves from his body were in the range of wavelength between $0.7 \mathrm{~mm}$ and 0.9.After passing through a focusing lens that acts as a filter, Images were sent to the computer processor system, then their location was determined. Considering the Received wavelength of any point of the body, a certain color is shown as an image. The Detection accuracy of the emission radiation from the skin depends on the specialist's skills in reading thermography images and Operator expertise in setting the vision window. The scale of the selected color and the distance between patient and camera are inter-dependent phenomena. Initially, 60 patients suspected of breast tissue disease were studied by the help of thermography, ultrasound and biopsy. Before performing the Thermal imaging cases such as: Conditions of Imaging, temperature of room, comfort of the patient in room, Priority of performed thermography were taken into account before ultrasound because these factors were instrumental in providing the correct or false answer. Afterwads, the patients were asked to complete a questionnaire including their age, weight, medications, completion of time periods, and history of breast cancer. After clarifying these cases, thermography tests were performed and the researchers requested the patiants that their upper body cloths be disrobed completely from the body. Then the patient was allowed to rest for moments in order to let his body gain thermal equilibrium. Finally, the patient raised their hands. Then the operator provided thermography images from the patients in 3 directions. It is noteworthy that, to reduce the stress in patients for imaging which could affect on their blood flow and the temperature of the skin, stages of work were explained to the patients, and we assured them that no one travels during the test. After thermal imaging, the patients were guided to the biopsy and ultrasound section.

\section{Results}

In this study, after receiving and recording the patient's history, the results of the clinical examination along with the ultrasound and biopsy stereotypes and images of thermography were examined separately by the respective specialists. After recording the results, and considering the clinical symptoms, Capabilities of thermography

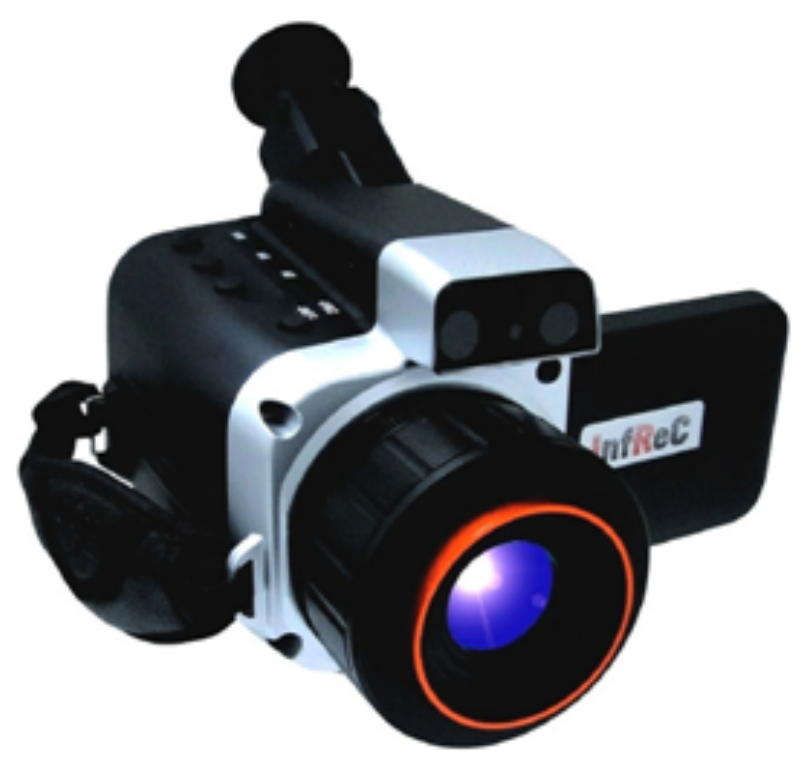

Figure 1. Figure of thermography device R500 (taken from device) 
techniques were compared in identifying whether the case was healthy or not. In general, 60 patients whose ages were 44/9 years, while, the lowest one was 21 and the maximum age was 73 years old . Figure 2 is an example of the analysis of asymmetry on the photo taken from the patient.With respect to the results of ultrasound and pathology; the patient had a hypoechoic mass in the left breast with a diameter of $19 * 9$.The Average temperature in the area $\mathrm{A}$ was $35.42^{\circ}$ and in the area $\mathrm{B}$ was $33.99^{\circ} \mathrm{C}$. The maximum temperature in the area $\mathrm{A}$ is $35.99^{\circ} \mathrm{C}$ and

Table 1. Displays the Characteristics Related to Noncontact Infrared Camera INFREC R500 Model

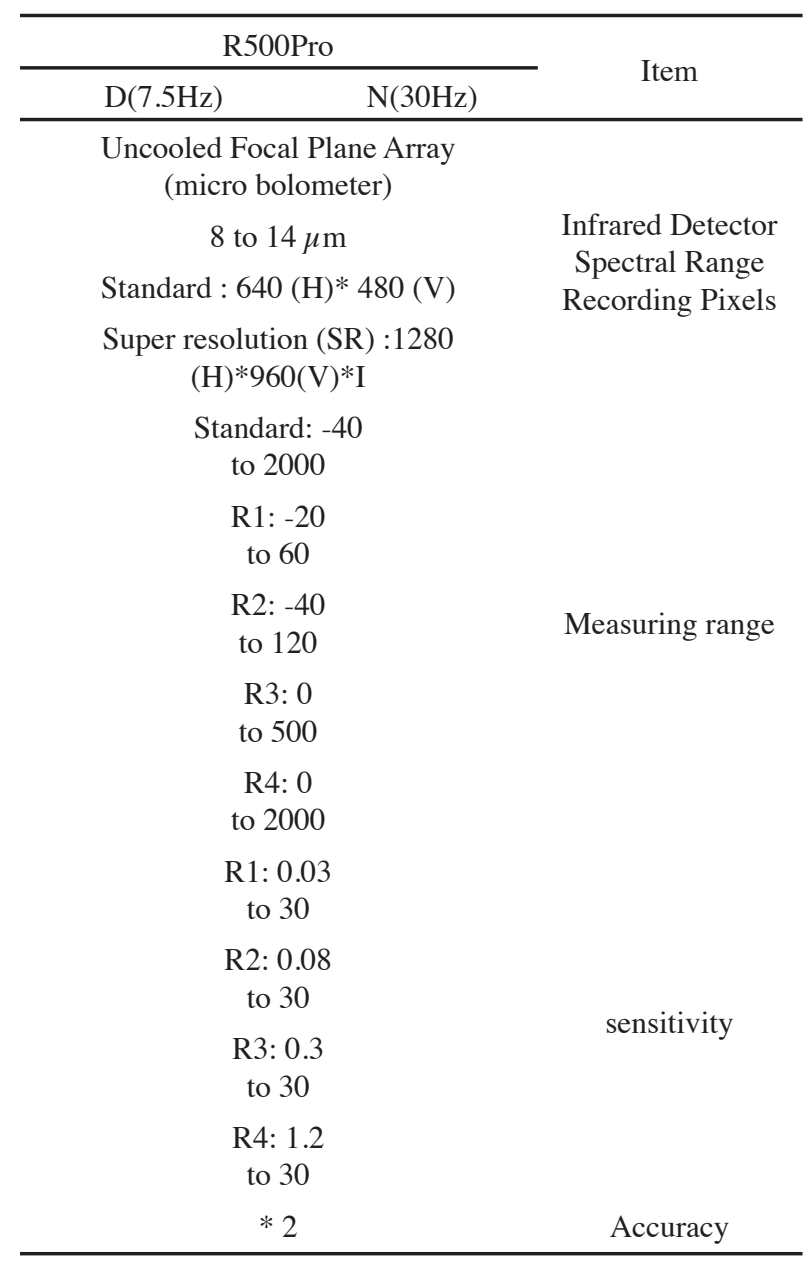

in the area $\mathrm{B}$ is equal to $34.68^{\circ} \mathrm{C}$. Regarding the abovementioned matters, the asymmetry was determined clearly in two areas.

Table 2 shows the results of thermography related to patients compared with pathology. One of the factors discussed in this research is the subject of asymmetry in thermography. If this factor is detected correctly, it will help the screening and diagnosing of the exact location of the cancer or diseases related to the breast tissue. The existence of these factors convinced us that there are complications in breast tissue which must be used in other methods for identifying the exact type of complication. One of the factors which has been addressed in this research is the case of asymmetry in thermography.

In the remaining 7 patients, case complications were observed. 3 patients suffered Heterogeneous mass, fibroadenoma, Intraductal masses. In which Asymmetries and the exact location of the problems were not detected properly. But in 2 cases, fibromyalgia, fibro Glandular and spycoleh masses were observed.Finally, in 2 cases, asymmetry and the exact location of the tumor was not detected, which included ISO-echo with cystic and Calcified mass. Additionally, results were analyzed in terms of temperature. According to the magnitude of

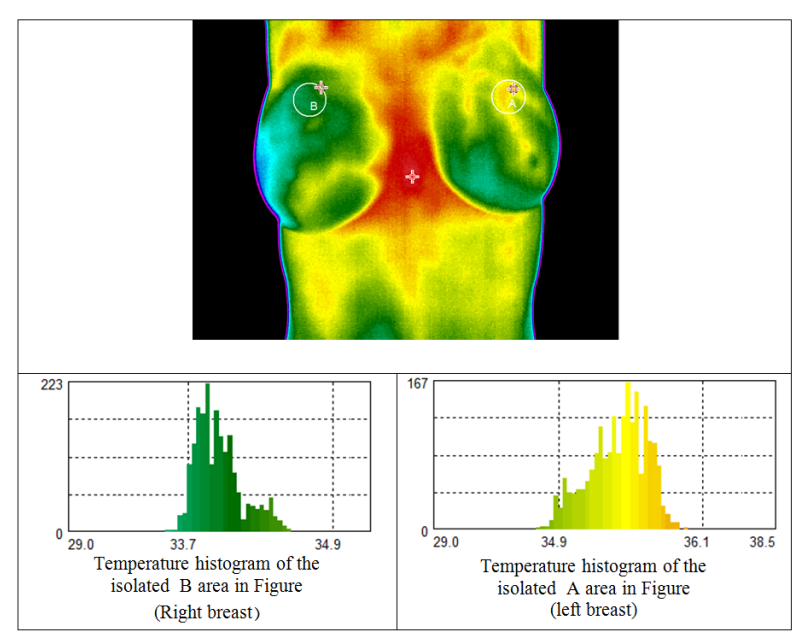

Figure 2. Temperature Histogram of two Separated Area in the left and Right Breast

Table 2. The Results of Thermography Testing Based on the Type of the Tumor

\begin{tabular}{ccccc}
\hline Symptomatic & samples & Asymmetry & $\begin{array}{c}\text { Asymmetry in subjects without } \\
\text { single breast }\end{array}$ & $\begin{array}{c}\text { Diagnosing the exact location } \\
\text { of the disturbed area }\end{array}$ \\
\hline hypoechoic mass & 39 & $87.17 \%$ & $91.89 \%$ & $61.53 \%$ \\
Cystic mass & 14 & $85.71 \%$ & $92.30 \%$ & $75 \%$ \\
\hline
\end{tabular}

Table 3. Sensitivity of IR imaging by age

\begin{tabular}{cccccc}
\hline Characteristic & samples & Asymmetry & $\begin{array}{c}\text { Asymmetry in subjects } \\
\text { without single breast }\end{array}$ & $\begin{array}{c}\text { Diagnosing the exact location } \\
\text { of the disturbed area }\end{array}$ \\
\hline \multirow{2}{*}{ Age } & $30-40$ & 8 & $87 \%$ & $87 \%$ & $75 \%$ \\
& $40-50$ & 14 & $100 \%$ & $100 \%$ & $64 \%$ \\
\hline
\end{tabular}


Table 4. Classifying the subject samples based on symptom types and their standard Thermobiological scores

\begin{tabular}{|c|c|c|c|c|c|c|}
\hline Symptom & $\begin{array}{c}\text { Number of } \\
\text { samples }\end{array}$ & TH1 & TH2 & TH3 & TH4 & TH5 \\
\hline Hypoechoic mass & 39 & 1 & 2 & 3 & - & 33 \\
\hline Cystic mass & 14 & - & - & 2 & - & 12 \\
\hline Heterogeneous, Fibroadenomas, Ayntradktal mass & 3 & - & - & - & - & 2 \\
\hline Fibroglandular and Spiculated mass & 2 & - & - & - & - & 2 \\
\hline ISO echo with cystic and calcified mass & 2 & 2 & - & - & - & - \\
\hline
\end{tabular}

temperature changes, all images were divided into 2 classes; normal $(\Delta \mathrm{T}<1)$, abnormal $(\Delta \mathrm{T}>1)$.Out of the 60 females screened, $\Delta \mathrm{T}<1$ was observed for 9 cases ( 3 cases had single breast). Out of these 51 subjects had $(\Delta T>1)$. The sensitivity of IR imaging by tumor, age, size is shown in Table 3 . Regarding the results, it is clear that the higher is the age, the more difficult is the cancer detection. The analyzed images can be classified based on the Thermobiological scores into five main groups: TH1-Normal image without vascularity (blood vessels), TH2-Normal image with vascularity, TH3-Equivocal (questionable, but not abnormal), TH4-Abnormal TH 5-Very abnormal. Table 4 presents the results of classifying thermal images on 60 patients based on their Thermobiological scores. It is observed that asymmetry and thermal class are directly related to each other.

\section{Discussion}

Though thermography imaging has its own limitations in terms of sensitivity and specificity and it is dependent on experiment qualification, it provides important information about the physiological situation of the breast (Moghbel and Mashohor, 2013). Unfortunatly, The risk of getting breast cancer has tripled since the bygone half century with respect to modification of lifestyle and other factors (Long and Beales, 2014). Consequently, it is essential to detect breast cancer beforehand to better administrator this growing lethal menace. For decades, Sonography and mammography have been used as the screening methods of select (Penhoet et al., 2005).

The risk of breast cancer during the past half century has tripled due to the changes in the people lifestyle and some other factors. (Muffazzal et al., 2014). The significance of this study is due to the fact that there has been no comprehensive review and comparison done in diagnosing the diseases of breast tissue to relate thermography and ultrasound. Findings from the study indicate that thermography has either advantages or disadvantages in the detection of breast disease. With the advent of a new generation of infrared detectors, infrared thermal imaging has become a medical diagnostic tool for measuring the thermal pattern of abnormal areas. Furthermore, features of Thermal imaging include Sensitivity to the temperature, spatial resolution, and no contact, safe. Thermal images can be stored digitally and then processed by using various software packages and the researcher obtained a comprehensive understanding from the thermal model. The results of this study show that thermography can be used for the early detection and rapid screening. To complete this procedure, methods such as ultrasound can be employed. It can also be considered as a complementary method for ultrasound procedure. The next point is that asymmetry plays a key role in the early detection that we can recognize by means of the basic settings of the camera.similarly, Thermography is suitable compared to the ultrasound diagnosis in detecting the breast tissue diseases such as cystic masses and hypoecho masses by adopting asymmetry technique. But this method has some drawbacks in detecting the exact location of the masse, such as cystic. However, thermography is weak in the asymmetry technique and detection of the exact location related to masses as micro calcification. And important point is that, people who once suffered from cancer and had the contents of their infected breast tissue evacuated, the use of asymmetry techniques would be inefficient in thermography.

\section{Acknowledgements}

We are thankful for this research has been supported by advanced diagnostic and interventional radiology research center of Tehran University of medical science grant 27466-98-04-93 on Tuesday, January 20, 2015.

\section{References}

Acharya UR, Ng EYK, Tan JH, et al (2012). Thermography based breast cancer detection using texture features and support vector machine. J Med Systems, 36, 1503-10.

Araujo MC, Lima RC, De Souza RM (2014). Interval symbolic feature extraction for thermography breast cancer detection. Expert Systems Applications, 41, 6728-37.

Arora N, Martins D, Ruggerio D, et al (2008). Effectiveness of a noninvasive digital infrared thermal imaging system in the detection of breast cancer. Am J Surg, 196, 523-6.

Collett AE, Guilfoyle C, Gracely EJ, et al (2014). Infrared imaging does not predict the presence of malignancy in patients with suspicious radiologic breast abnormalities. Breast J, 20, 375-80.

Kelly KM, Dean J, Comulada WS, et al (2010). Breast cancer detection using automated whole breast ultrasound and mammography in radiographically dense breasts. Eur Radiol, 20, 734-42.

Lahiri B, Bagavathiappan S, Jayakumar T, et al (2012). Medical applications of infrared thermography: a review. Infrared Phys Technol, 55, 221-35.

Long E, Beales IL (2014). The role of obesity in oesophageal cancer development. Therapeutic Advances Gastroenterol, 7, 247-68.

Moghbel M, Mashohor S (2013). A review of computer assisted 
detection/diagnosis (CAD) in breast thermography for breast cancer detection. Artificial Intelligence Review, 39, 305-13.

Muffazzal R, Poonam M, Rajkumar M, et al (2014). Evaluation of digital infrared thermal imaging as an adjunctive screening method for breast carcinoma: A pilot study. Int J Surg, 12, 1439-43.

$\mathrm{Ng}$ EK (2009). A review of thermography as promising noninvasive detection modality for breast tumor. Int $J$ Thermal Sci, 48, 849-59.

Nicandro CR, Efren MM, Maria Yaneli AA, et al (2013). Evaluation of the diagnostic power of thermography in breast cancer using bayesian network classifiers. Computational and mathematical methods in medicine.

Penhoet EE, Petitti DB, Joy JE (2005). Saving Women's lives: strategies for improving breast cancer detection and diagnosis, National Academies Press.

Saika K, Sobue T (2009). Epidemiology of breast cancer in Japan and the US. JMAJ, 52, 39-44.

Steketee J (1973). Spectral emissivity of skin and pericardium. Physics Med Biol, 18, 686.

Vreugdenburg TD, Willis CD, Mundy L, et al (2013). A systematic review of elastography, electrical impedance scanning, and digital infrared thermography for breast cancer screening and diagnosis. Breast Cancer Res Treat, 137, 665-76.

Watmough D, Fowler PW, Oliver R (1970). The thermal scanning of a curved isothermal surface: implications for clinical thermography. Physics Med Biol, 15, 1.

Wishart G, Campisi M, Boswell M, et al (2010). The accuracy of digital infrared imaging for breast cancer detection in women undergoing breast biopsy. Eur J Surg Oncol, 36, 535-40. 